PROCEEDINGS OF THE

AMERICAN MATHEMATICAL SOCIETY

Volume 48, Number 1, March 1975

\title{
MACDONALD IDENTITIES AND EUCLIDEAN LIE ALGEBRAS
}

\author{
R. V. MOODY ${ }^{1}$
}

ABSTRACT. The Macdonald identities on affine root systems are placed in the context of Euclidean Lie algebras. This yields a simplified form of the identities, which is used to infer several results on the partition function of the root system of a Euclidean Lie algebra.

Introduction. In a remarkable paper [7], Macdonald showed that from each reduced affine root system one is able to produce an identity in the formal exponentials of roots of this system, and that the se identities are generalizations of a classical identity of Weyl for finite root systems and certain identities connected with Dedekind's $\eta$-function.

A feature of the Macdonald identities (see equation (M) of $\$ 2$ ) is the appearance of a factor $P^{*}$ whose description is quite awkward and whose meaning is very obscure. Our intention here is to show that it is possible to place the identities in the context of Euclidean Lie algebras, whereupon the meaning of $P^{*}$ becomes obvious and the identities take on a simpler and even more beautiful appearance.

In their new form, the identities give a marvellous relationship between the Weyl group, the root system, and the dimensions of the root spaces. It is not unrea sonable to expect that similar identities may hold for all the Lie algebras determined by arbitrary Cartan matrices. Extensive computer calculations by Dr. K. L. Teo give strong evidence to support this. To be precise, we conjecture that Proposition 2 remains true when $\Omega$ is any reduced heffalump Lie a lgebra.

In $\$ 3$ we use the new form of the identity to generalize some well-known results on Kostant's partition function to partition functions on the Euclidean root systems.

Received by the editors November 13, 1973.

AMS (MOS) subject classifications (1970). Primary 17B65, 17B35, 17B15, $10 A 20$.

${ }^{1}$ The author gratefully acknowledges the support of a National Research Council of Canada grant while this work was being done.

Copyright ( 1975, American Mathematical Society 
1. Heffalump Lie algebras. Let $\mathbf{Z}, \mathbf{N}, \mathbf{Z}^{+}$denote the integers, the natural numbers, and the nonnegative integers, respectively. Let $K$ be a field of characteristic zero, and let $\left(A_{i j}\right)$ be an $l \times l$ matrix of integers such that (i) $A_{i i}=2, i=1, \cdots, l$, (ii) $A_{i j} \leq 0$ if $i \neq j$, (iii) $A_{i j}=0$ if and only if $A_{j i}=0$. We call such a matrix a Cartan matrix. The Cartan matrices occuring classically in the classification of finite-dimensional semisimple Lie algebras will be called Cartan matrices of finite type. The concept of indecomposable is defined in the usual way [6, p. 130]. We assume from now on that our Cartan matrices are indecomposable. Apart from the class of finite type matrices, there is another special class called the Euclidean matrices. These are defined in $\$ 2(i)$. Any Cartan matrix which is neither finite nor Euclidean will be called ultra-affine.

The Lie algebra $\tilde{\Omega}$ over $K$ defined by generators $e_{1}, \cdots, e_{l}, f_{1}, \cdots, f_{l}$, $h_{1}, \cdots, h_{l}$ and relations $\left[h_{i} h_{j}\right]=0,\left[e_{i} h_{j}\right]=A_{i j} e_{i},\left[f_{i} h_{j}\right]=-A_{i j} f_{i},\left[e_{i} f_{j}\right]=$ $\delta_{i j} h_{i} \forall i, j$; and $e_{i}\left(\operatorname{ad} e_{j}\right)^{-A_{i j}+1}=0, f_{i}\left(\operatorname{ad} f_{i}\right)^{-A_{i j}+1}=0 \forall i, j$ with $i \neq j$, is called the standard heffalump'Lie a lgebra defined by $\left(A_{i j}\right)$ over $K$. The basic properties of $\tilde{\Omega}$ have been derived in [3], [2]. The subspace $H=$ $K h_{1}+\cdots+K h_{l}$ is an abelian subalgebra. $\tilde{\Omega}$ is graded by $A \equiv \mathbf{Z} \alpha_{1}+\cdots+$ $\mathbf{Z} \alpha_{l}$, where $\alpha_{1}, \cdots, \alpha_{l}$ are $\mathbf{Z}$-free generators. The $\beta \in A$ for which the corresponding homogeneous space $\tilde{\Omega}_{\beta}$ is not zero form a set $\tilde{\Delta}$ called the root system of $\tilde{\Omega}$. Every root $\beta$ is either a nonnegative or a nonpositive integral combination of $a_{1}, \cdots, a_{l}$; and $\tilde{\Delta}=\tilde{\Delta}^{+} \cup\{0\} \cup \tilde{\Delta}^{-}$, where $\tilde{\Delta}^{+}$and $\tilde{\Delta}^{-}$are the positive and negative roots, respectively, and $\tilde{\Delta}^{-}=-\tilde{\Delta}^{+}$. Let $A_{K}$ denote $K \otimes_{\mathrm{Z}} A$. There is a homomorphism $\epsilon: A_{K} \rightarrow \widetilde{H}^{*}$, and for $\alpha \in \tilde{\Delta}$, $x \in \tilde{\Omega}_{a}, b \in \tilde{H}$, we have $[x h]=(\epsilon(a))(b) x$. For simplicity we write $a(b)$ for $(\epsilon(a))(b)$.

Let $\widetilde{U}$ be the universal enveloping algebra of $\tilde{\Omega}$. $\tilde{\Omega}$ is a $\widetilde{U}$ module via the adjoint action. The radical $R$ of $\tilde{\Omega}$ is the $\widetilde{U}$ module generated by all the homogeneous $x \in \tilde{\Omega}$ for which $x \widetilde{U} \neq \tilde{\Omega}$. $\tilde{\Omega} / R$ is called the reduced heffalump a lgebra defined by $\left(A_{i j}\right)$. It is similarly graded by $A$ and has a root system $\Delta=\Delta^{+} \cup\{0\} \cup \Delta^{-}$. In fact one can show that $\Delta=\tilde{\Delta}$, but we will not need this.

Let $H$ be the image of $\tilde{H}$ under $\tilde{\Omega} \rightarrow\{$. The mapping $\epsilon$ induces a linear mapping, also denoted $\epsilon$, from $A_{K}$ into $H^{*}$. For each $j \in\{1, \cdots, l\}$ the linear endomorphism of $A_{k}$ defined by $\alpha_{i} r_{j}=\alpha_{i}-A_{i j} \alpha_{j}$ is an involution and the 
group $W$ generated by the $r_{i}$ is a Coxeter group. We have $\Delta W=\Delta$. The set $\Delta_{W} \equiv\left\{\alpha_{1}, \cdots, \alpha_{l}\right\} W \subset \Delta$ is the set of real or Weyl roots. We have $\Delta_{W}=$ $\Delta-\{0\} \Leftrightarrow\left(A_{i j}\right)$ is of finite type $\Leftrightarrow \operatorname{dim} \Omega<\infty \Leftrightarrow \operatorname{dim} \tilde{\Omega}<\infty$ [1]. For $\alpha \in \Delta_{W}$, $\operatorname{dim} \Omega_{a}=1$. The set $\Delta_{I} \equiv \Delta-\Delta_{W}$ is called the set of imaginary roots. $A$ root $\beta \in \Delta^{+}$is real if and only if there is a $w \in W$ such that $\beta w \in \Delta^{-}$. Thus the elements of $\Delta_{I}^{+} \equiv \Delta_{I} \cap \Delta^{+}$are permuted amongst themselves by $W$.

For each $w \in W$, we denote by $l(w)$ the reduced length of $w$ : i.e. the minimum integer $k$ such that $w$ can be expressed as a product of $k$ of the generating involutions $r_{1}, \cdots, r_{l}$. By convention, $l(1)=0$. We let $N(w)=$ $\left\{a \in \Delta^{+}: \alpha w \in \Delta^{-}\right\}$. As in [5], card $N(w)=l(w)$. Finally we set $s(w)=$ $\Sigma\left\{\alpha: \alpha \in N\left(w^{-1}\right)\right\}$. Note that $s(w)$ is a sum of a finite number of positive real roots.

2. Macdonald's identity for Euclidean Lie algebras. Let $\left(A_{i j}\right)$ be a Euclidean matrix. It is convenient here to suppose that our indices run from 0 to $l$. Such a matrix may be characterized by either of the two properties:

(a) $\left(A_{i j}\right)$ is an indecomposable Cartan matrix which is singular, and each of its submatrices obtained by deleting one row and corresponding column is a Cartan matrix (not necessarily connected) of finite type.

(b) $\left(A_{i j}\right)$ is an indecomposable Cartan matrix, and there is a set $d_{i}, i=$ $0, \cdots, l$, of nonnegative integers, not all zero, such that $\Sigma d_{i} \alpha_{i}$ acts as the zero functional on $\widetilde{H}[3]$.

The following facts are known about $\{$ and $\Delta$ [9].

(i) The map $A_{K} \stackrel{\epsilon}{\rightarrow} H^{*}$ is surjective and has a one-dimensional kernel $K \xi$ where $\xi$ is some positive root $\xi=\Sigma_{i=0}^{l} \xi_{i} \alpha_{i}$, where each $\xi_{i} \in \mathbf{N}$, and $\left(\xi_{0}, \cdots, \xi_{l}\right)=1$.

(ii) $\mathrm{Z} \xi \subset \Delta$ and $\Delta=\Delta_{W} \cup \mathrm{Z} \xi$ (disjoint union).

(iii) If $\alpha \in \Delta$ and $\alpha+k \xi \in \Delta$, then $\alpha+\mathbf{Z} k \xi \subset \Delta$. There are always some roots (even fundamental roots) $\alpha$ such that $\alpha+\xi \in \Delta$. This is not true for all $\alpha$ in general. However, there is a number $r$ (tier number) which is 1,2 , or 3 such that $\alpha \in \Delta \Rightarrow \alpha+r \xi \in \Delta$.

(iv) $\tilde{\Omega}$ has a nontrivial radical $R$ which is the center and $\tilde{\Omega}_{0} \cap R$ is one dimensional. Thus $H$ has dimension $l$.

(v) There is a symmetric bilinear form $(\cdot, \cdot)$ on $A_{K}$ relative to which $2\left(\alpha_{i}, \alpha_{j}\right) /\left(\alpha_{j}, \alpha_{j}\right)=A_{i j}$ and moreover, for all $\alpha, \beta \in \Delta_{W}, 2(\alpha, \beta) /(\beta, \beta) \in \mathbf{Z}$. $(\cdot, \cdot)$ is positive semidefinite and its radical is $K \xi$. Since $K \xi=\operatorname{Ker} \epsilon, H^{*}$ 
inherits a positive definite form. For $\alpha \in \Delta_{W}, \beta \in A, \beta r_{\alpha}=\beta-$ $2(\beta, \alpha) /(\alpha, \alpha) \alpha$.

(vi) $\epsilon\left(\Delta_{W}\right)$ is a finite root system in $H^{*}$.

(vii) It is possible to index the fundamental roots $\alpha_{0}, \cdots, \alpha_{l}$ in such a way that (a) $\xi_{0}=1$, (b) $\alpha_{0}$ is a terminal vertex of the Dynkin diagram of $\left(A_{i j}\right),(\mathrm{c})$ (only used if (a) and (b) fail to characterize a root to within symmetries of the diagram) $\alpha_{0}+\xi \in \Delta$.

(viii) Putting $m_{\alpha}=\operatorname{dim} \mathfrak{R}_{\alpha}$ we have always $m_{\alpha}=m_{\alpha+k r \xi} \forall k \in \mathbf{Z}$. If $r>1$ and $I_{1} \equiv\left\{i: 1 \leq i \leq l\right.$ and $\left.\alpha_{i}+\xi \in \Delta\right\}$, then $m_{\xi}=\operatorname{card} I_{1}$ and $m_{k \xi}=$ $m_{\xi} \forall k$ with $k \equiv 0(\bmod r)$. On the other hand, $\forall k \equiv 0(\bmod r), m_{k \xi}=l=$ $\operatorname{card}\{1, \cdots, l\}$. For $i \in I_{2} \equiv\{1, \cdots, l\}-I_{1}, \alpha_{i}+s \xi \in \Delta \Leftrightarrow s \equiv 0(\bmod r)$. This result can be read off Table 2 in [9]. If $r=1$ and $I_{1}, I_{2}$ are defined as above, then $I_{1}=\{1, \cdots, l\}, I_{2}=\phi$, and $m_{k \xi}=\operatorname{card} I_{1} \forall k$. Note that the index 0 does not appear in these statements.

We use $\Delta$ to make an affine root system as defined by Macdonald in [7]. We use the set $H$ as the underlying affine space with the vector space $H$ as its group of translations. The unique positive definite form on $H$ inherited from $H^{*}$ determines the metric. Let $F$ be the space of affine linear functionals on $H \cdot \operatorname{dim} F=\operatorname{dim} H+1=l+1$.

Define $\pi: A_{K} \rightarrow K$ to be the 0 th projection map $\Sigma c_{i} a_{i} \mapsto c_{0}$. Define $f: A_{K} \rightarrow F, \alpha \mapsto f_{\alpha}$, by $f_{\alpha}(x)=\pi(\alpha)+\alpha(x) \forall x \in H, \forall \alpha \in A_{K} \cdot f_{\alpha}$ is a $n$ affine linear functional, and its derivative $D f_{\alpha} \in H^{*}$ is $\epsilon(\alpha)$. Clearly $f$ is linear. Furthermore, $f_{\alpha}=0 \Rightarrow \pi(\alpha)+\alpha(x)=0 \forall x \in H \Rightarrow \pi(\alpha)=0$ and $\alpha(x)=0 \forall x \in H$ $\Rightarrow \alpha \in K \xi$ and $\pi(\alpha)=0 \Rightarrow \alpha=0$. Comparing dimensions, we see that $f$ is an isomorphism. Let $S=f\left(\Delta_{W}\right)$. We propose to show that $S$ is an irreducible affine root system.

1. The elements of $S \operatorname{span} F$ and are nonconstant functions: It is clear that since $f$ is an isomorphis $m$ and $\alpha_{0}, \cdots, \alpha_{l}$ span $A_{K}, S$ spans $F$. In addition, $\alpha \in \Delta_{W} \Rightarrow \epsilon(\alpha) \neq 0 \Rightarrow f_{\alpha}$ not constant.

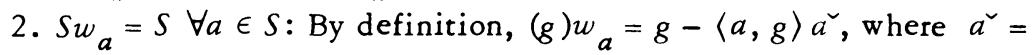
$2 a /\langle a, a\rangle$ and $\langle\cdot, \cdot\rangle$ is defined as follows: $\langle a, b\rangle=\langle D a, D b\rangle$, where $\langle\cdot, \cdot\rangle$ is defined on $H^{*}$ by the form on $H$. Since $H$ has the form inherited by $H^{*}$, the form $\langle\cdot, \cdot\rangle$ on $H^{*}$ is just the inherited form from $A_{K}$. Thus suppose $a \in S$ and $a=f_{\alpha}$. Then for any $g=f_{\beta}, \beta \in A_{K}$,

$$
g w_{a}=f_{\beta}-\langle\alpha, \beta\rangle 2 f_{\alpha} /\langle\alpha, \alpha\rangle=f(\beta-2\langle\alpha, \beta\rangle /\langle\alpha, \alpha\rangle \alpha)=f\left(\beta r_{\alpha}\right) .
$$

Consequently, $\left(f_{\beta}\right) w_{a}=f_{\beta r_{a}}$. In particular, if $\beta \in \Delta_{W}$, so does $\beta r_{a}$, whence $f_{\beta r_{\alpha}} \in S$. 
3. $\left\langle a, b^{\sim}\right\rangle \in \mathbf{Z}$ for all $a, b \in S:$ Clear from above.

For $a \in F, a$ not constant, let $H_{a}$ denote the affine hyperplane $\{x \in H$ : $a(x)=0\}$. If $a=f_{a}$ we write $H_{a}$ for $H_{a}$.

4. The group, $W(S)$, of affine isometries generated by the reflections $w_{a}$ in the hyperplanes $H_{a}, a \in S$, acts properly on $H$ : First note from the proof of 3, that the map $r_{\alpha} \mapsto w_{f_{\alpha}}$ determines an isomorphism of $W$ onto $W(S)$ by which we may identify the two groups, so that $f$ is a $W$-isomorphism. Furthermore, for $w \in W(S), g \in F, x \in H$, we have $g(x w)=\left(g w^{-1}\right)(x)$.

Now we use an argument made available to us by R. Rasala [11]-namely, if $C$ is a nonempty open subset of $H$ such that $\forall w \in W, C w \cap C \neq \varnothing \Rightarrow w=$ 1 , then $W$ acts properly on $H$. Indeed, suppose such a $C$ exists and let $r>0$ be chosen so that the open ball $B_{r}(x)$ of radius $r$ about $x$ lies in $C$. We can assume that $C=B_{r}(x)$. The $W$ translates of $C$ are all disjoint. For any two sets $X, Y$ lying in $H$, let $[X, Y]$ denote $\{w \in W: X w \cap Y \neq \varnothing\}$. Suppose now that $X$ and $Y$ are compact. We have to show that $[X, Y]$ is finite. Choose $s \geq r$ such that $X, Y \subseteq \overline{B_{s}(x)}$. Then $[X, Y] \subseteq\left[\overline{B_{s}(x)}, \overline{B_{s}(x)}\right]$. If $w \in\left[\overline{B_{s}(x)}\right.$, $\overline{\left.B_{s}(x)\right]}$, then $\overline{B_{s}(x w)} \cap \overline{B_{s}(x)} \neq \varnothing$, whence $d(x, x w) \leq 2 s$ and $\overline{B_{s}(x w)} \subseteq$ $\overline{B_{3 s}(x)}$. Then

$$
C w=B_{r}(x) w=B_{r}(x w) \subseteq \overline{B_{s}(x w)} \subseteq \overline{B_{3 s}(x)} .
$$

The last set being compact, the number of such $w$ is finite.

Let $D_{i}$ be the open half-space determined by $H_{a_{i}}$ and containing $h_{i}, i=$ $0,1, \cdots, l$. We apply the above argument to $C \equiv \bigcap_{i=0}^{l} D_{i}$. Note that $x \in C$ $\Leftrightarrow f_{a_{i}}(x)>0 \forall i=0,1, \cdots, l$ since $f_{a_{i}}\left(b_{i}\right)>0$. It is easy to see that $C \neq \varnothing$. Now if $w \in W, w \neq 1$, then $a_{i} w^{-1} \in \Delta^{-}$for at least one $i$ and, hence,

$$
f_{a_{i}}(x w)=\left(f_{a_{i}} w^{-1}\right)(x)=f_{a_{i} w^{-1}}(x)<0,
$$

and $x u \notin C$. Thus $C w \cap C=\varnothing$.

Since $\left(A_{i j}\right)$ is indecomposable, it is clear that the affine roots $S$ cannot be decomposed into two disjoint sets of mutually orthogonal roots. Thus $S$ is irreducible.

Proposition 1. The set $C$ defined above is a chamber with $H_{a_{0}}, H_{a_{1}}, \cdots$, $H_{a_{l}}$ as its walls. The set $\left\{f_{a_{0}}, f_{a_{1}}, \cdots, f_{a_{l}}\right\}$ is the corresponding base. The set $\left\{\epsilon \alpha_{1}, \cdots, \epsilon \alpha_{l}\right\}$ is a base for the gradient root system $\Sigma$ of $S$.

Proof. To show that $C$ is a chamber, it will suffice to show that no 
hyperplane $H_{\beta}, \beta \in \Delta_{W}$, can meet $C$. Let $x \in C$ and $\beta=\sum_{i=0}^{l} z_{i} a_{i} \in \Delta_{W}$. We may assume that $\beta \in \Delta^{+}$. For $i=1, \cdots, l, f_{\alpha_{i}}(x)=\alpha_{i}(x)>0$, while $f_{a_{0}}(x)=$ $1+\alpha_{0}(x)>0$. Thus

$$
f_{\beta}(x)=z_{0}+\sum_{i=0}^{l} z_{i} a_{i}(x)=z_{0}+z_{0} a_{0}(x)+\sum_{i=1}^{l} z_{i} a_{i}(x)>0,
$$

whence $x \notin H_{\beta}$. It is clear that the $H_{a_{i}}$ are the walls, and $\left\{f_{a_{0}}, \cdots, f_{\alpha_{l}}\right\}$ is the base by definition.

Next we show $\alpha_{1}, \cdots, \alpha_{l}$ form a base for the gradient root system. Let $x_{0}$ be the vertex $H_{a_{1}} \cap \cdots \cap H_{a_{l}}$. Since $\sum_{i=0}^{l} \xi_{i} \alpha_{i}=0$ and $\xi_{0}=1$, it suffices by [7, Proposition 6.6] to prove that $x_{0}$ is special for $W(S)$. Then Macdonald's proof actually shows $\alpha_{1}, \cdots, \alpha_{l}$ form a base of $\Sigma$. Now $x_{0}$ is special for $W(S)$ means that if $H_{\beta}$ is any hyperplane, $\beta \in \Delta_{W}$, then some parallel hyperplane $H_{\gamma}$ passes through $x_{0}$. We have $x_{0} \in H_{\gamma} \Leftrightarrow f_{\gamma}\left(x_{0}\right)=0$. Let $\gamma=\sum_{i=0}^{l} c_{i} a_{i}$. Then

$$
f_{\gamma}\left(x_{0}\right)=\sum c_{i} f_{a_{i}}\left(x_{0}\right)=c_{0} f_{a_{0}}\left(x_{0}\right)=c_{0}+a_{0}\left(x_{0}\right)=\pi(\gamma)+a_{0}\left(x_{0}\right) .
$$

However, since $\epsilon \Sigma \xi_{i} a_{i}=0$, we have $\Sigma \xi_{i} \alpha_{i}\left(x_{0}\right)=0$, so $\alpha_{0}\left(x_{0}\right)=0$. Thus $f_{\gamma}\left(x_{0}\right)=\pi(\gamma)$. We conclude that all the hyperplanes $H_{\gamma}$ such that $\pi(\gamma)=0$ pass through $x_{0}$. Clearly any hyperplane $H_{\beta}$ is parallel to one of these.

It is clear that the Dynkin diagram for $f_{a_{0}}, \cdots, f_{\alpha_{l}}$ coincides with the diagram for $a_{0}, \cdots, \alpha_{l}$ in $\Delta$. It is also clear that under $f$ the positive roots $\Delta_{W}^{+}$coincide with the positive roots $S^{+}$relative to $f_{a_{0}}, \cdots, f_{a_{l}}$.

Let $F_{0}$ be the 1-dimensional space of constant functions on $H$. We have $f(K \xi)=F_{0}$ since $f_{k \xi}(x)=k+k \xi(x)=k$. Thus $c$, the unique positive generator of $L(S) \cap F_{0}$, corresponds to $\xi$ itself $(L(S)$ is the lattice generated by $S)$. For each $a \in S, u_{a} \in \mathbf{N}$ is defined as the smallest positive integer such that $a+u_{a} c \in S$ (Macdonald's $u_{a}$ is our $u_{a} c$ ). Thus, if $a=f_{a}, u_{a}$ is the smallest positive integer such that $\alpha+u_{a} \xi \in \Delta$. There is no harm in writing $u_{a}$ for $u_{a}$.

Let $\Gamma$ denote the group algebra of $A$. In $\Gamma, A$ appears multiplicatively, and $a \in A$ is denoted by $e(\alpha)$. Here, as elsewhere in this paper, we will be dealing with infinite sums and products of elements of $\Gamma$. In all cases these are of a formal nature and there is no problem about the meaning. Similarly, we let $\Gamma^{*}$ denote the group algebra of the lattice $L(S)$.

Macdonald's identity states that in $\Gamma^{*}$ : 


$$
\sum_{w \in W(S)}(-1)^{l(w)} e(-s(w))=P^{*} \prod_{a \in S^{+}}(1-e(-\alpha))
$$

where

$$
P^{*}=\prod_{n=1}^{\infty} \prod_{\alpha \in B(\Sigma)}\left(1-e\left(-n u_{\alpha} c\right)\right),
$$

and $B(\Sigma)$ is any base of the gradient root system.

Since $L(S) \cong A$ and $f$ is a $W$-mapping, this identity is equivalent to the identity in $\Gamma$ :

$$
\sum_{w \in W}(-1)^{l(w)} e(-s(w))=P \prod_{a \in \Delta_{W}^{+}}(1-e(-\alpha)),
$$

where

$$
P=\prod_{n=1}^{\infty} \prod_{a \in B(\Sigma)}\left(1-e\left(-n u_{\alpha} \xi\right)\right) .
$$

Since $\alpha_{1}, \cdots, \alpha_{l}$ form a base for the gradient root system,

$$
P=\prod_{n=1}^{\infty} \prod_{i=1}^{l}\left(1-e\left(-n u_{a_{i}} \xi\right)\right) .
$$

Here, $u_{\alpha_{i}}$ is the least positive integer such that $\alpha_{i}+u_{\alpha_{i}} \xi \in \Delta$.

Let $I_{1}$ and $I_{2}$ be defined as in (viii) above. Then

$$
\begin{aligned}
\prod_{n=1}^{\infty} \prod_{i=1}^{l}\left(1-e\left(-n u_{a_{i}} \xi\right)\right) & =\prod_{n=1}^{\infty}\left(\prod_{I_{1}}(1-e(-n \xi)) \prod_{I_{2}}(1-e(-n r \xi))\right) \\
& =\prod_{n=1 ; n \neq 0(\bmod r)} \prod_{I_{1}}(1-e(-n \xi)) \prod_{n=1}^{\infty} \prod_{I_{1}}(1-e(-n r \xi)) \\
& \cdot \prod_{n=1}^{\infty} \prod_{I_{2}}(1-e(-n r \xi)) \\
= & \prod_{n=1 ; n \neq 0(\bmod r)}^{\infty}(1-e(-n \xi))^{m} \xi \prod_{n=1}^{\infty}(1-e(-n r \xi))^{l} \\
= & \prod_{n=1 ; n \neq 0(\bmod r)}^{\infty}(1-e(-n \xi))^{m} n \xi \prod_{n=1 ; n \equiv 0(\bmod r)}^{(1-e(n \xi))^{m} n \xi} \\
& =\prod_{n=1}^{\infty}\left(1-e(-n \xi)^{m} n \xi .\right.
\end{aligned}
$$


Combining this with the rest of the product, and using the facts that $m_{\alpha}=1$ if $\alpha \in \Delta_{W}$ and $\Delta^{+}=\Delta_{W}^{+} \cup \mathbf{N} \xi$, we have

Proposition 2. Let $\{$ be a Euclidean Lie algebra with root system $\Delta$ and Weyl group $W$. Let $m_{\alpha}=\operatorname{dim} \Omega_{a}$. Then in the group algebra of the group $A$ generated by the roots,

$$
\sum_{w \in W}(-1)^{l(w)} e(-s(w))=\prod_{a \in \Delta^{+}}(1-e(-\alpha))^{m_{a}} .
$$

3. Partition functions. Let $\Delta$ be the root system of a Euclidean Lie algebra. Let $\Lambda=\left\{\gamma_{i}: i \in \mathbf{N}\right\}$ be a set of symbols, and let ${ }^{*}: \Lambda \rightarrow \Delta^{+}$be a surjective mapping such that for each $\alpha \in \Delta^{+}$, the complete pre-image of ${ }^{*}$ in $\Lambda$ has precisely $m_{a}$ elements. We define the Kostant partition function $P: A \rightarrow \mathrm{Z}^{+}$by

$$
P(\beta)=\operatorname{card}\left\{f: \Lambda \rightarrow \mathbf{Z}^{+}: \sum_{i=1}^{\infty} f\left(\gamma_{i}\right) \gamma_{i}^{*}=\beta\right\},
$$

and the partition function into distinct parts $P_{0}: A \rightarrow \mathrm{Z}^{+}$by

$$
P_{0}(\beta)=\operatorname{card}\left\{f: \Lambda \rightarrow\{0,1\}: \sum_{i=1}^{\infty} f\left(\gamma_{i}\right) \gamma_{i}^{*}=\beta\right\}
$$

These functions are independent of the choice of $\Lambda$ and take the value zero on all $\beta \notin \mathbf{Z}^{+} \alpha_{0}+\cdots+\mathbf{Z}^{+} \alpha_{l}$. If $f: \Lambda \quad\{0,1\}$ is one of those defining $P_{0}(\beta)$, we say $f$ is even if $\Sigma_{i=1}^{\infty} f\left(\gamma_{i}\right)$ is even and let $P_{0}^{+}(\beta)$ denote the number of such functions. Similarly we define $P_{0}^{-}(\beta)$. Note that

$$
\sum_{\beta \in A} P(\beta) e(\beta)=\prod_{\beta \in A}(1-e(\alpha))^{-m} \alpha
$$

and

$$
\prod_{\beta \in A}(1-e(\alpha))^{m} a=\sum_{\beta \in A}\left(P_{0}^{+}(\beta)-P_{0}^{-}(\beta)\right) e(\beta) .
$$

Proposition 3. (i) $P_{0}^{+}(\beta)=P_{0}^{-}(\beta)$ if $\beta \notin\{s(w): w \in W\}$;

(ii) for $\beta=s(w), P_{0}^{+}(\beta)=1$ or 0 according as $l(w)$ is even or odd, and $P_{0}^{-}(\beta)=0$ or 1 according as $l(w)$ is even or odd.

Proof. (i) follows from Proposition 2, where the involution $e(\beta) \mapsto$ $e(-\beta)$ has been applied to the identity. 
(ii) It is a straightforward induction on $l(w)$ to see that for $w=r_{i_{k}} \cdots$ $r_{i}$ (reduced),

$$
s(w)=\alpha_{i_{1}}+\alpha_{i_{2}} r_{i_{1}}+\cdots+\alpha_{i_{k}} r_{i_{k-1}} \cdots r_{i_{1}},
$$

and this is the unique way of expressing $s(w)$ as a sum of positive roots.

Proposition 4. For $a \in A$,

$$
\sum_{w \in W}(-1)^{l(w)} P(\alpha-s(w))=\delta_{a, 0}
$$

where $\delta$ is Kronecker's $\delta$.

Proof. This follows directly from

$$
\begin{aligned}
1 & =\sum_{\beta \in A} P(\beta) e(\beta) \cdot \prod_{\alpha \in \Delta^{+}}(1-e(\alpha))^{m} \alpha \\
& =\sum_{\beta \in A} P(\beta) e(\beta) \cdot \sum_{w \in W}(-1)^{l(w)} e(s(w)) .
\end{aligned}
$$

This result is especially interesting because if we let $U$ denote the universal enveloping algebra of $\Sigma_{a \in \Delta^{+}} \Omega_{a}$, then $U$ is graded naturally by $A$, and $\operatorname{dim} U_{\alpha}=P(\alpha) \forall \alpha$.

Added in proof. V. G. Kac [Infinite-dimensional Lie algebras and Dedekind's $\eta$-functions, Funkcional. Anal. i Priložen. 8 (1974), no. 1, 77-78] has proved the conjecture mentioned in the introduction under the assumption that $\left(A_{i j}\right)$ is symmetrizable [8]. In particular this work establishes the Macdonald identities (in the form (M)) by techniques which are intrinsically related to the corresponding Lie algebras.

\section{REFERENCES}

1. S. Berman, Thesis, Indiana University, Bloomington, Ind., 1971. $158-183$.

2. - On the construction of simple Lie algebras, J. Algebra 27 (1973),

3. S. Berman, R. Moody and M. Wonenburger, Cartan matrices with null roots and finite Cartan matrices, Indiana Univ. Math. J. 21 (1971/72), 1091-1099. MR $45 \# 3439$.

4. N. Bourbaki, Éléments de mathématique. Fasc. XXXIV. Groupes et algèbres de Lie. Chaps. 4, 5, 6, Actualités Sci. Indust., no. 1337, Hermann, Paris, 1968. MR 39 \#1590.

5. N. Iwahori, On the structure of a Hecke ring of a Chevalley group over a finite field, J. Fac. Sci. Univ. Tokyo Sect. I 10 (1964), 215-236. MR 29 \#2307.

6. N. Jacobson, Lie algebras, Interscience Tracts in Pure and Appl. Math., no. 10, Interscience, New York, 1962. MR $26 \# 1345$. 
7. I. G. Macdonald, Affine root systems and Dedekind's $\eta$-function, Invent. Math. 15 (1972), 91-143.

8. R. Moody, A new class of Lie algebras, J. Algebra 10 (1968), 211-230. MR 37 \#5261.

9. - Euclidean Lie algebras, Canad. J. Math. 21 (1969), 1432-1454. MR $41 \# 287$.

10. R. Moody and K. L. Teo, Tits' systems with crystallographic Weyl groups, J. Algebra 21 (1972), 178-190.

11. R. Rasala, Notes on Coxeter groups (unpublished).

DEPARTMENT OF MATHEMATICS, UNIVERSITY OF SASKATCHEWAN, SASKATOON, SASKATCHEWAN, CANADA 